\title{
Motif based hierarchical random graphs: structural properties and critical points of an Ising model*
}

\author{
Monika Kotorowiczli, Yuri Kozitsky \\ Institute of Mathematics, Maria Curie-Skłodowska University, Lublin, Poland
}

Received March 15, 2010, in final form June 7, 2010

\begin{abstract}
A class of random graphs is introduced and studied. The graphs are constructed in an algorithmic way from five motifs which were found in [Milo R., Shen-Orr S., Itzkovitz S., Kashtan N., Chklovskii D., Alon U., Science, 2002, 298, 824-827]. The construction scheme resembles that used in [Hinczewski M., A. Nihat Berker, Phys. Rev. E, 2006, 73, 066126], according to which the short-range bonds are non-random, whereas the long-range bonds appear independently with the same probability. A number of structural properties of the graphs have been described, among which there are degree distributions, clustering, amenability, small-world property. For one of the motifs, the critical point of the Ising model defined on the corresponding graph has been studied.
\end{abstract}

Key words: amenability, degree distribution, clustering, small-world graph, Ising model, critical point

PACS: 89.75.Fb, 89.75.Kd, 05.10.Cc, 05.70.Jk

\section{Introduction and setup}

A vast variety of large systems occurring in nature and society have a very complicated topological structure. These are the Internet, the World Wide Web, citation, neural, and social networks, etc. In view of the complex topology and unknown organizing principles, the networks are often modeled as random graphs. A random graph with a given node set $V$ is a graph in which for a given pair $i, j \in V$, the bond $\langle i, j\rangle$ appears at random. The study of random graphs has been originated by P. Erdős and A. Rényi, who were the first to introduce such graphs in [1, 2]. In the Erdős-Rényi random graph model, denoted by $G_{n, p}$, the number of nodes is $n$, and the bonds between distinct nodes appear independently 1 with the same probability $p$. An important characteristic of a graph is the node degree, which is the number of bonds attached thereat. In $G_{n, p}$, it is a random variable, and all such variables are independent and have the same probability distribution. Namely, the probability that the degree of a given node is $k$ is given by the Bernoulli law

$$
P_{n}(k)=\left(\begin{array}{c}
n-1 \\
k
\end{array}\right) p^{k}(1-p)^{n-1-k}, \quad k=0,1, \ldots, n-1 .
$$

For random graphs, the most interesting questions refer to their asymptotic properties in the limit $n \rightarrow+\infty$. To get nontrivial answers to such questions one allows the parameters to depend on $n$. For $p_{n}=c / n$, the limit of (1) is the Poisson law

$$
P(k)=c^{k} e^{-c} / k !, \quad k \in \mathbb{N}_{0} .
$$

However, for irregular complex networks, the random graph $G_{n, p}$ is not a good model since in the most of such networks the degree distribution is essentially non-Poissonian. Many real world

\footnotetext{
*This work was supported by the DFG through the project 436 POL $125 / 0-1$ as well as through SFB 701: "Spektrale Strukturen und Topologische Methoden in der Mathematik". Yuri Kozitsky was also supported by TODEQ MTKD-CT-2005-030042.

†E-mail: monika@hektor.umcs.lublin.pl

‡E-mail: jkozi@hektor.umcs.lublin.pl

${ }^{1}$ C.f., however, the discussion in [3].
} 
networks, e.g. the WWW, are characterized by power-law node degree distributions, which have the form $P(k)=C k^{-\gamma}, \gamma>1$, typical for the so-called scale-free graphs.

Another important parameter characterizing a random graph is the clustering coefficient, which is the probability that two nodes are neighbors given they have a common neighbor. Clearly, in $G_{n, p}$ this probability is $p$, and is the same independently of whether or not the nodes have a common neighbor. Real world networks usually manifest strong clustering, which once more indicates that Erdős-Rényi type random graphs are not appropriate as their models. In [4], D.J. Watts and H. Strogatz proposed another type of random graphs, in which the mentioned disadvantage is overcome. It should be noted, however, that such graphs do not have power law node degree distributions. The next step beyond the Erdős-Rényi model was done by A.-L. Barabási and R. Albert in [5]. In their model, the preferential attachment principle has been employed, typical for many real networks (the Internet, citation and social networks). According to this principle, the more connected a node is, the more likely it receives a new bond. The construction of the BarabásiAlbert model starts from an initial graph with $m_{0} \geqslant 2$ nodes (neither can be isolated). At each step, one adds a new node and connects it to the existing nodes. The probability $p_{i}$ that the new node is connected to node $i$ is proportional to the degree $n_{i}$ of that node, that is, $p_{i}=n_{i} /\left(\sum_{j} n_{j}\right)$.

Many of the complex networks occurring in nature contain characteristic patterns, recurring much more frequently than the other ones. They are called network motifs, see [6 11]. Different networks may have different motifs, and motifs in turn can characterize the networks. For instance, in biological regulation networks it has been experimentally demonstrated that each of the motifs can perform a key information processing function, see [8]. In [10], the authors introduced a random graph model based on some geometric principles (constraints). Then, they compared the appearance of eight elementary three- and four-node patterns in their model with the same characteristics of the Erdös-Rényi random graph. It turned out that five of these patterns are motifs for their model, but not for the Erdős-Rényi random graph, see figure 1 below and table 1 in 10 . For
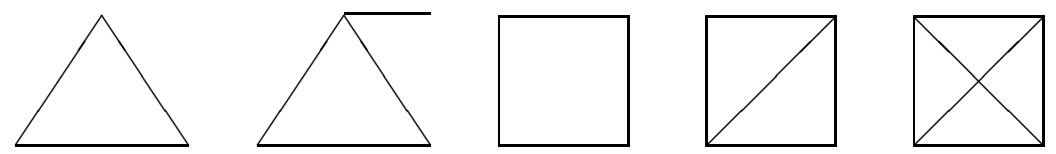

Figure 1. Three and four node motifs $M_{1}, M_{2}, M_{3}, M_{4}, M_{5}$ found in [10].

another random graph model, the appearance of the same eight patterns was also studied in [11]. Note that among these patterns, only $M_{1}$ and $M_{5}$ correspond to complete graphs (each node is a neighbor to every other node).

One of the ways to get information about infinite graphs, also random ones, is to study the properties of certain models of statistical physics defined thereon. The most popular ones are the Ising and Potts models, as well as the models of bond and site percolation, see [12]. On the other hand, in statistical physics certain graphs are employed to mimic a crystal lattice, for which the critical-point behavior of the Ising model can be described in an explicit and rigorous way. These are the so-called hierarchical lattices introduced in [13]. Such lattices are defined in a rather algorithmic way by means of the basic pattern, e.g., by a "diamond", which is the pattern $M_{3}$ depicted in figure 1. A mathematical description of the Gibbs states of the Ising model on such graphs was done by P.M. Bleher and E. Žalys in [14, 15]. M. Hinczewski and A. Nihat Berker [16] studied the critical-point properties of the Ising model on the diamond hierarchical lattice 'decorated' by additional bonds, which appear at random. In the present paper, we follow the way suggested in [16] and introduce hierarchical graphs constructed by means of the motifs shown in figure 1, decorated by additional bonds which appear at random and repeat, in a way, the corresponding motif. We analyse some of their characteristics, such as the average degree, the node degree distribution, amenability, the small-world property, as well as the critical-point properties of the Ising model. This study of ours is a continuation of [17], where the graph based on $M_{1}$ was introduced by one of us. Note that our hierarchical graphs also found applications in cryptography, see [18]. 


\section{The graphs: construction and structural properties}

\subsection{The construction: informal description}

As is typical for hierarchical graphs, e.g., for hierarchical lattices in [13, 16], the construction is carried out in an algorithmic way: at $k$-th level, $k \in \mathbb{N}$, one produces a subgraph, say $\Lambda_{k}$, which is then used as a construction element for producing $\Lambda_{k+1}$. The procedure is the same at each level, and the starting element is obtained from the corresponding motif. Let us illustrate this for the simplest case based on the motif $M_{1}$. First we label the nodes of $M_{1}$ by $a, b$, and $c$, as it is shown in figure 2. and obtain $\Lambda_{1}$ - the triangle. Then we take three such graphs and label them by the same labels. As a result, each of the triangles has nodes of two kinds: one node the label of which coincides with the triangle label (e.g., node $a$ in triangle $a$ ), and two nodes with non-coinciding labels. Thereafter, the triangles are glued up according to the following rule: node $c$ of triangle $a$ is glued up with node $a$ of triangle $c$, ect. The nodes with the coinciding labels remain untouched. These are the so-called external nodes of $\Lambda_{2}$. The remaining nodes are called internal. The bonds of the initial triangles turn into the bonds of $\Lambda_{2}$. We call them basic bonds; they are depicted by solid lines. At the next stage of the first step, we add the bonds connecting the external nodes in the same way as it is in the motif $M_{1}$. Such bonds are depicted by dotted lines and are called decorations. As a result, we obtain the graph $\Lambda_{2}$, which has six basic bonds and three decorations, three external and three internal nodes. Then we repeat the same procedure - take three copies of $\Lambda_{2}$, label them by $a, b$, and $c$, and divide their external nine nodes into two groups: three nodes with coinciding labels and six nodes with non-coinciding labels. Then the graphs $\Lambda_{2}$ are glued up as described above. Thereafter, three decorating bonds are drawn to connect the external nodes. This procedure is repeated ad infinitum. Similar constructions for motifs $M_{2}$ and $M_{3}$ are presented in figure 3 and figure 4, respectively. Here we have omitted the decorating bonds not to overload the pictures. The picture for $M_{4}$ is obtained from that for $M_{3}$ by adding the diagonals. The picture for $M_{5}$ is just the three-dimensional version of the picture for $M_{1}$, where the basic pattern is a tetrahedron.

\subsection{Definitions}

In order to fix the terminology and to make the construction of our graphs mathematically immaculate, we begin by introducing a number of relevant mathematical notions. A (simple) graph $\mathrm{G}$ is a pair of sets $(\mathrm{V}, \mathrm{E})$, where $\mathrm{V}$ is the set of nodes, whereas $\mathrm{E}$ is a subset of the Cartesian product $\mathrm{V} \times \mathrm{V}$. We suppose that $\mathrm{E}$ is symmetric and irreflexive, i.e., $\langle j, i\rangle \in \mathrm{E}$ whenever $\langle i, j\rangle \in \mathrm{E}$, and $\langle i, i\rangle \notin \mathrm{E}$ for every $i, j \in \mathrm{V}$. We say that $i$ and $j$ are connected by a bond if $\langle i, j\rangle \in \mathrm{E}$. In this case, we write $i \sim j$ and say that $i$ and $j$ are adjacent or that they are neighbors. Hence, the elements of $\mathrm{E}$ themselves can be called bonds. The graph is said to be complete if each two nodes are adjacent. For a given $i$, by $n(i)$ we denote the degree of $i$ - the number of its neighbors. If $\mathrm{V}$ is finite, then $\mathrm{E}$ is also finite, and the graph is said to be finite. Otherwise, the graph is infinite. An infinite graph is called locally finite, if $n(i)$ is finite for every node. All the infinite graphs we study are countable, which means that both sets $\mathrm{V}$ and $\mathrm{E}$ are infinite and countable. Given $\mathrm{G}=(\mathrm{V}, \mathrm{E})$ and $\mathrm{G}^{\prime}=\left(\mathrm{V}^{\prime}, \mathrm{E}^{\prime}\right)$, let $\phi: \mathrm{V} \rightarrow \mathrm{V}^{\prime}$ be such that $\phi(i) \sim \phi(j)$ whenever $i \sim j$. Such a map $\phi$ is called a morphism. A bijective morphism is called an isomorphism. If $\phi$ is an isomorphism, then its inverse $\phi^{-1}$ is also an isomorphism, and then the graphs $G$ and $G^{\prime}$ are said to be mutually isomorphic. Such graphs have identical structures and thus can be identified. In this case, we also say that $\mathrm{G}^{\prime}$ is a copy of $\mathrm{G}$. One observes that this refers to both finite and infinite graphs. An isomorphism $\phi: \mathrm{V} \rightarrow \mathrm{V}$, i.e. which maps the graph onto itself, is called an automorphism. The graph $\mathrm{G}^{\prime}=\left(\mathrm{V}^{\prime}, \mathrm{E}^{\prime}\right)$ such that $\mathrm{V}^{\prime} \subset \mathrm{V}$ and $\mathrm{E}^{\prime} \subset \mathrm{E}$ is said to be a subgraph of $\mathrm{G}=(\mathrm{V}, \mathrm{E})$. In this case, we write $\mathrm{G}^{\prime} \subset \mathrm{G}$. Suppose that a subgraph $G^{\prime} \subset G$ has a copy, say $\widetilde{G}$, that is, there exists an isomorphism $\phi: \widetilde{G} \rightarrow G^{\prime}$. Then $\phi$, considered as a map $\phi: \widetilde{G} \rightarrow G$, is called an embedding of $\widetilde{G}$ into $G$, whereas $G^{\prime}$ is called the image of $\widetilde{\mathrm{G}}$ under this embedding. Figure 1 presents the so-called unlabeled graphs, which we call patterns. After labeling, i.e., attaching a label to each of the nodes, such a pattern turns into a graph. Another labeling may or may not yield the same graph. This depends on whether or not 
there exists the corresponding automorphism. For instance, any labeling of the triangle $M_{1}$ yields the same graph since in any case each of the nodes has the same neighbors. For the pattern $M_{2}$, the left-hand graph in figure 3 with the interchanged labels $a$ and $b$ is the same. However, the graph with the interchanges $c$ and $d$ is not the same anymore. Of course, this new graph is isomorphic to the initial one. This is because there is only one nontrivial automorphism of $M_{2}$ : the one which interchanges $a$ and $b$, and preserves $c$ and $d$. The triangle has six automorphisms.

Let us now turn to random graphs. To introduce such a graph we need an underlying graph $\mathrm{G}=(\mathrm{V}, \mathrm{E})$ and a family $\mathcal{E}$ of subsets of $\mathrm{E}$. If $\mathrm{G}$ is finite, as $\mathcal{E}$ one can take the set of all subsets of $E$. In the sequel, we deal with such graphs only. Thus, for $E^{\prime} \in \mathcal{E}$, we say that $E^{\prime}$ has been picked at random with probability $P\left(\mathrm{E}^{\prime}\right)$. In the Erdős-Rényi model $G_{n, p}$, the underlying graph is complete with the node set $\mathrm{V}=\{1, \ldots, n\}$. In this model, $P\left(\mathrm{E}^{\prime}\right)=p^{\left|\mathrm{E}^{\prime}\right|}$, where $p \in[0,1]$ and $\left|E^{\prime}\right|$ stands for the number of elements in $E^{\prime}$. In other words, the elements of $E$ are being picked independently, each with the same probability $p$. In a bit complicated model, the bonds are picked independently but with probability which depends on the bond. In this case, as well as in the case of the Erdős-Rényi model, we deal with a random graph with independent bonds. For such graphs,

$$
P\left(\mathrm{E}^{\prime}\right)=\prod_{e \in \mathrm{E}^{\prime}} p(e)
$$

where $p(e)$ is the probability of picking bond $e$. The set of graphs $\mathrm{G}^{\prime}=\left(\mathrm{V}, \mathrm{E}^{\prime}\right)$ with $\mathrm{E}^{\prime} \in \mathcal{E}$ is called the graph ensemble - each $\mathrm{G}^{\prime}$ is picked at random from this ensemble. A random graph model is the pair consisting of the graph ensemble and of the function $P: \mathcal{E} \rightarrow[0,1]$. If the function $P$ is as in (3), the graph is said to be a random graph with independent bonds. Suppose that we have two random graph models with independent bonds. Let also $G=(V, E)$ and $\widetilde{G}=(\widetilde{V}, \widetilde{E})$ be their underlying graphs and $\phi: \mathrm{V} \rightarrow \widetilde{\mathrm{V}}$ be a morphism. Then this map is said to be the morphism of the random graphs if for every $\langle i, j\rangle \in \mathrm{E}$, the probability (in the first model) that this bond is picked is the same as the corresponding probability (in the second model) for the bond $\langle\phi(i), \phi(j)\rangle$.

\subsection{The construction}

As was mentioned above, each of our graphs is constructed in an algorithmic way from the corresponding motif presented in figure 1, Since they are going to be random graphs with independent bonds, we have to construct the corresponding underlying graphs and, to define, for a given bond, the probability of being picked, c.f. (3). In all our models, the bonds will be of two kinds, which we call basic bonds and decorations. Basic bonds are going to be non-random, i.e. they are picked with probability one. Decorating bonds appear with probability $p \in[0,1]$, which is a parameter of the model. Turn now to the construction of the underlying graphs. Let $q$ be the number of nodes in the corresponding motif, that is, $q=3$ for $M_{1}$ and $q=4$ for the remaining motifs. At step $k=1$, we just label the vertices of the corresponding motif by $i=1, \ldots, q$ and obtain the initial graph $\Lambda_{1}=\left(V_{1}, E_{1}\right)$. All its bonds are set to be basic. Suppose now that we have $q+1$ copies of $\Lambda_{1}$ obtained by the isomorphisms $\phi_{2}^{j}, j=0,1, \ldots, q$. Thus, in $j$-th copy the nodes are $\phi_{2}^{j}(i)$, $i=1, \ldots, q$. The graph $\Lambda_{2}$ is obtained from these copies under the following conditions

$$
\phi_{2}^{0}(i)=\phi_{2}^{i}(i), \quad i=1, \ldots, q ; \quad \phi_{2}^{i}(j)=\phi_{2}^{j}(i), \quad i=1, \ldots, q, i \neq j .
$$

Thus, the images of $V_{1}$ under $\phi_{2}^{i}$ and $\phi_{2}^{j}$ with $i \neq j$ intersect only at one node where (4) holds. The maps $\phi_{2}^{j}, j=0,1, \ldots, q$ embed $\Lambda_{1}$ into $\Lambda_{2}$. The nodes $\phi_{2}^{i}(i), i=1, \ldots, q$, are called the external nodes of $\Lambda_{2}$. All other nodes are called internal. Thus, $\Lambda_{2}$ has $q$ external and $q(q-1) / 2$ internal nodes. At this stage, we label them by $i=1, \ldots, q(q+1) / 2$ in such a way that the external nodes have the same labels as in $\Lambda_{1}$, that is, $\phi_{2}^{i}(i)=i, i=1, \ldots q$. By construction, the bonds obtained as images under the map $\phi_{2}^{0}$ are decorations: they are of the form $\left\langle\phi_{2}^{0}(i), \phi_{2}^{0}(j)\right\rangle$ where $i$ and $j$ are adjacent in $\Lambda_{1}$. From the first condition in (4) we see that the decorating bonds connect the external nodes of $\Lambda_{2}$. The remaining bonds of $\Lambda_{2}$ are set to be basic. Now we construct $\Lambda_{3}$ from one copy of $\Lambda_{1}$ and $q$ copies of $\Lambda_{2}$. Let $\phi_{3}^{0}$ be the map which produces the copy of $\Lambda_{1}$ and $\phi_{3}^{j}$, 
$j=1, \ldots, q$ be the maps which produce the copies of $\Lambda_{2}$. We then impose the conditions

$$
\phi_{3}^{0}(i)=\phi_{3}^{i}(i), \quad i=1, \ldots, q ; \quad \phi_{3}^{i}(j)=\phi_{3}^{j}(i), \quad i=1, \ldots, q, i \neq j
$$

and obtain $\Lambda_{3}$. Thus, $\phi_{3}^{0}$ embeds $\Lambda_{1} \rightarrow \Lambda_{3}$, and $\phi_{3}^{i}: \Lambda_{2} \rightarrow \Lambda_{3}, i=1,2, \ldots, q$. As above, the nodes $\phi_{3}^{i}(i)$ are set to be external, and the remaining nodes are internal. The images of $V_{2}$ under $\phi_{3}^{i}$ and $\phi_{3}^{3}$ with $i \neq j$ intersect only at one node where (5) holds. Again we label the nodes of $\Lambda_{3}$ in such a way that $\phi_{3}^{i}(i)=i, i=1, \ldots, q$. Now let us establish which bonds of $\Lambda_{2}$ are decorating and which are basic. As above, the bonds connecting the external nodes are decorating. The images of decorating bonds of $\Lambda_{2}$ are decorating bonds in $\Lambda_{3}$; the same is also true for the basic bonds - the basic bonds of $\Lambda_{3}$ are exactly the images of the basic bonds of $\Lambda_{2}$. For $k \geqslant 4$, the construction of $\Lambda_{k}$ from $\Lambda_{k-1}$ and $\Lambda_{1}$ is identical to the construction of $\Lambda_{3}$ just described. As above, by $V_{k}$ and $E_{k}$ we denote the sets of nodes and bonds of $\Lambda_{k}$, respectively. Thus, for $k \geqslant 2$ we have $E_{k}=E_{k}^{\prime} \cup E_{k}^{\prime \prime}$, where $E_{k}^{\prime}$ (respectively, $E_{k}^{\prime \prime}$ ) consists of basic (respectively, decorating) bonds. All $\Lambda_{k}, k \in \mathbb{N}$, are considered as subgraphs of an infinite graph $\Lambda_{\infty}$, the structure and properties of which are not important for the study presented in this article.

Note that the construction principle used above essentially differs from that used in the construction of the hierarchical lattices in 13 -16]. Namely, in our case to obtain $\Lambda_{k}$ one replaces each node of the basic pattern by a copy of the graph $\Lambda_{k-1}$. In the hierarchical lattices, one replaces a bond. As we shall see in the sequel, this leads to essentially different properties of the resulting graphs. Below in figure 2, we illustrate the construction described above for the case where the basic pattern is the motif $M_{1}$. In this case, the bare graph (i.e. the one which occurs for $p=0$ ) is the approximating graph for the fractal known as the Sierpiński gasket2. The elements of $E_{2}^{\prime}$ (middle graph) and of $E_{3}^{\prime}$ (right-hand graph) are depicted by solid lines. The elements of $E_{2}^{\prime \prime}$ and of $E_{3}^{\prime \prime}$ are depicted by dotted lines. We omit some dotted lines to indicate that they appear at random and hence may be absent in a given realization of the graph. Note that $\Lambda_{3}$ can be viewed as the triangle composed of three copies of $\Lambda_{2}$. In figure 3 we present the construction of the bare graph $\Lambda_{3}$ corresponding to $M_{2}$. In contrast to the former case, this is not a planar graph. In figure 4. we construct the bare graph $\Lambda_{2}$ for the motif $M_{3}$. One observes that in that picture the node $c$ of the lower left-hand quadrat (i.e. quadrat $a$ ) is glued up with node $a$ of the upper right-hand quadrat. It is interesting that the corresponding fractal can be obtained by the following procedure, resembling the one which yields the Sierpiński gasket. One takes the full quadrat and cuts it out into four equal quadrats, but without cutting the external lines. Then, one glues up the vertices of the smaller quadrats as depicted and proceeds with cutting out the smaller quadrats. The fractal which one obtains from $M_{5}$ is a three dimensional version of the Sierpiński gasket. One takes the full tetrahedron and cuts out its inner one fourth in such a way that the remaining four tetrahedra are glued up according to the rule: vertex $b$ of tetrahedron $a$ is glued up with vertex $a$ of tetrahedron $b$, etc.

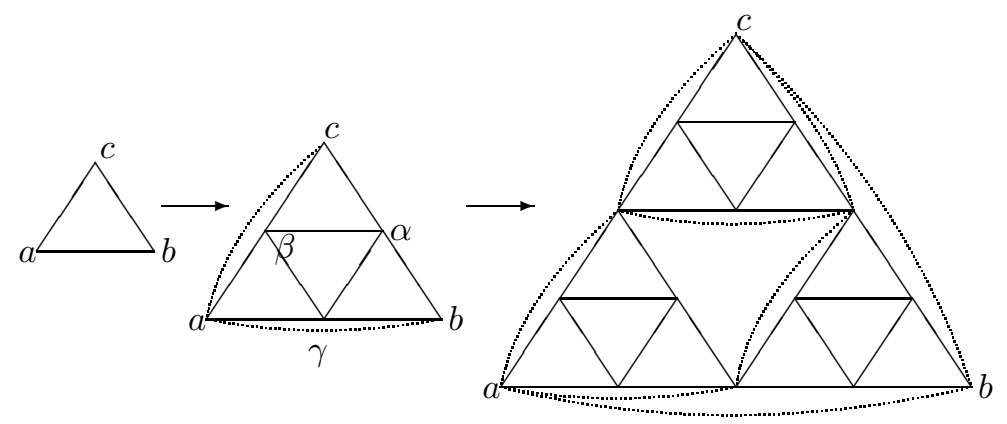

Figure 2. Construction of the graph $\Lambda_{3}$ based on $M_{1}$.

\footnotetext{
${ }^{2}$ The fractal itself is obtained as the closure of the set $\cup_{k \in \mathbb{N}} V_{k}$ in the appropriate topology, see e.g. [19].
} 


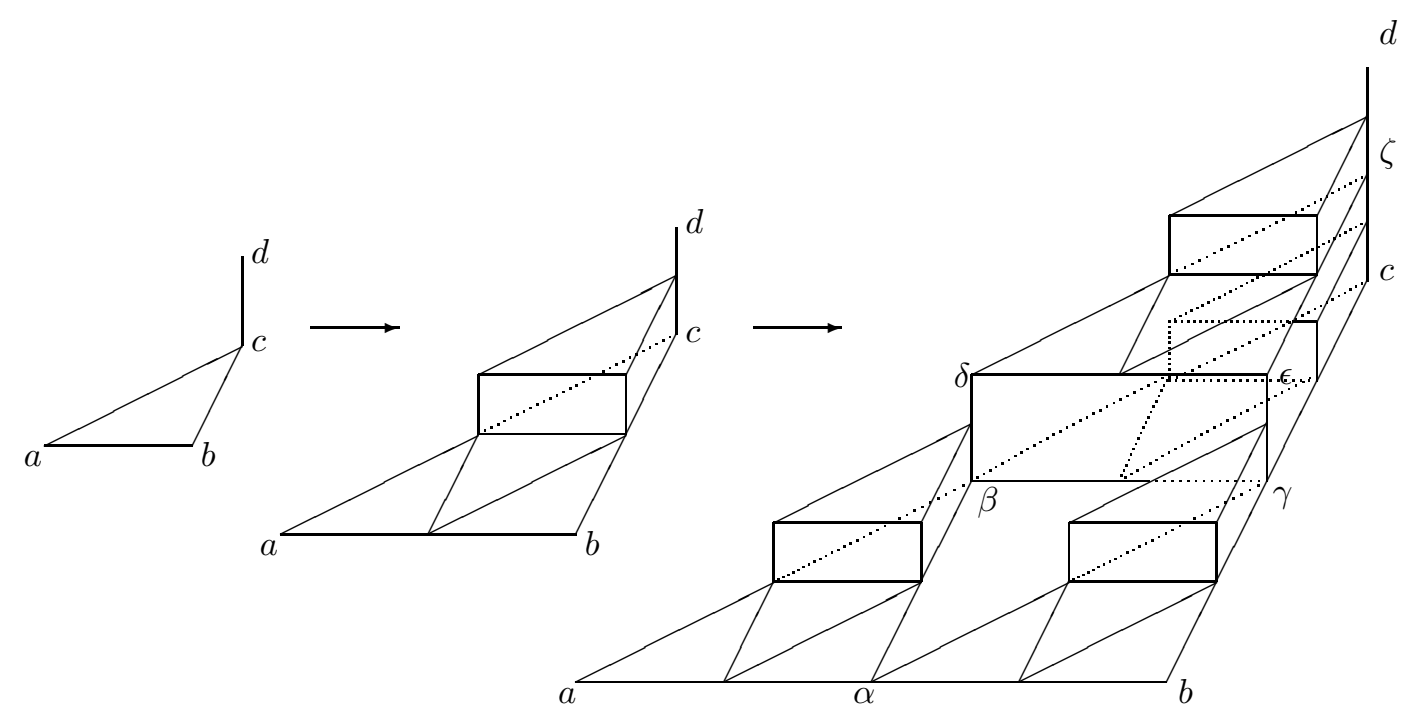

Figure 3. Construction of the bare graph $\Lambda_{3}$ based on $M_{2}$.

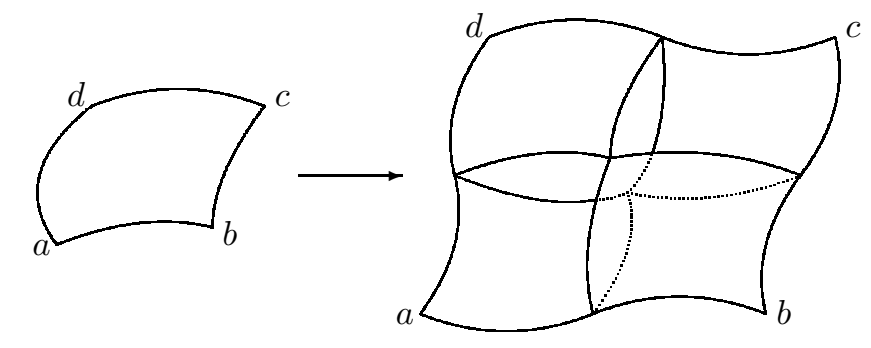

Figure 4. Construction of the bare graph $\Lambda_{2}$ based on $M_{3}$.

\subsection{Degree distribution}

Now we turn to the description of the structural properties of the graphs constructed above. Let $m_{k}, k \in \mathbb{N}$, be the number of times the basic pattern appears in non-decorated $\Lambda_{k}$ as a subgraph. For the graphs based on $M_{1}$ and $M_{5}$ we have the same situation. Here $m_{1}=1$ and $m_{k}=q m_{k-1}$, for $k \geqslant 2$ with the exception in $\Lambda_{2}$, where the additional pattern appears. So, for $M_{1}$ and $M_{5}$ we obtain $m_{1}=1$ and

$$
m_{k}=(q+1) q^{k-2}, \quad k \geqslant 2 .
$$

Here $q=3$ and $q=4$ for $M_{1}$ and $M_{5}$, respectively. For motif $M_{3}$ we have $m_{1}=1, m_{2}=2 q$ and

$$
m_{k}=q m_{k-1}, \quad k \geqslant 3 .
$$

Hence $m_{k}=2 \cdot 4^{k-1}$ for $k \geqslant 2$. The simplest case if for $M_{4}$, where $m_{k}=q m_{k-1}$ for $k \geqslant 2$. It gives $m_{4}=4^{k-1}$. The last motif is $M_{2}$ - triangle with additional bond. On each level this bond "produces" new 17 patterns. So $m_{1}=1$ and for $k \geqslant 2 m_{k}=\frac{1}{3}\left(26 \cdot 4^{k-1}-17\right)$.

Now we analyze the number of times the basic pattern appears in fully decorated $\Lambda_{k}$, denoted by $\widetilde{m}_{k}$. For the graphs based on $M_{1}$ and $M_{5}$ we have

$$
\widetilde{m}_{k}=\frac{2 q+1}{q-1} q^{k-1}+\frac{q+2}{q-1} .
$$

For $M_{3}$ it is $\frac{2}{3} 4^{k}-\frac{5}{3}$ and for $M_{4}$ we obtain $\frac{1}{3}\left(4^{k}-1\right)$. In all cases, we have $m_{k}$ increasing as $C(p) q^{k-1}$, which means that adding decorations does not change the asymptotics of $m_{k}$. 
In a similar way, we obtain

$$
\left|V_{k}\right|=\frac{q^{k}+q}{2}, \quad\left|E_{k}\right|=r q^{k-1}+r p \frac{q^{k-1}-1}{q-1}, \quad k \in \mathbb{N},
$$

where $\left|V_{k}\right|$ stands for the number of nodes in $\Lambda_{k}$, whereas $\left|E_{k}\right|$ is the expected number of bonds in this graph.

As was mentioned above, the degree distribution is a very important characteristic of the graph. In contrast to Erdös-Rényi type graphs, for our graphs the distribution of the random variable $n(i)$ depends on the type of $i$. Thus, the simplest way to describe this distribution is to average $n(i)$ over the nodes of a given $\Lambda_{k}$, that is, to consider

$$
n_{k}=\frac{1}{\left|V_{k}\right|} \sum_{i \in V_{k}} n(i)
$$

Let $\left\langle n_{k}\right\rangle$ be the expected value of $n_{k}$. Then

$$
\left\langle n_{k}\right\rangle=2\left|E_{k}\right| /\left|V_{k}\right|=\frac{4 r}{q(q-1)}(q-1+p)-\frac{4 r}{q(q-1)} \cdot \frac{q-1+2 p}{q^{k-1}+1} .
$$

However, this result provides only partial information on the node degree distribution. To get more, let us analyze the structure of the node sets $V_{k}, k=1,2, \ldots$, more in detail. For a given $\Lambda_{k}$ and $l=1, \ldots, k$, let $V_{k}^{(l)}$ be the set of nodes $i \in V_{k}$ which are external for some $\Lambda_{l}$ and, at the same time, are internal for any $\Lambda_{l+1}$. Of course, here we mean those $\Lambda_{l}$ 's which are subgraphs for $\Lambda_{k}$. As an example, let us consider the graph $\Lambda_{2}$ based on $M_{1}$, see the middle graph in figure 2 , The nodes $a, b$, and $c$ constitute $V_{2}^{(2)}$, whereas the remaining nodes constitute $V_{2}^{(1)}$.

The elements of $V_{k}^{(k-1)}$ are exactly the nodes at which the subgraphs $\Lambda_{k-1}^{j}, j=1, \ldots, q$ are glued up to form $\Lambda_{k}$, whereas the elements of $V_{k}^{(k)}$ are exactly the external nodes of $\Lambda_{k}$. Then $\left|V_{k}^{(k)}\right|=q$ and $\left|V_{k}^{(k-1)}\right|=q(q-1) / 2$. For $l<k-1$, we have $\left|V_{k}^{(l)}\right|=q\left|V_{k-1}^{(l)}\right|$, which can be solved to yield

$$
\left|V_{k}^{(l)}\right|=\frac{1}{2} q^{k-l}(q-1), \quad l=1, \ldots, k-1, \quad\left|V_{k}^{(k)}\right|=q .
$$

The reason to consider the sets $V_{k}^{(l)}$ is that all the elements of each such $V_{k}^{(l)}$ have the same degree distribution, independent of $k$ for $l \leqslant k-1$. In fact, the degrees of $i \in V_{k}^{(1)}$ are non-random since these nodes receive no decorating bonds. For such $i, n(i)=\sum_{j} n^{(0)}(j)$, where $n^{(0)}(j)$ is the degree of the corresponding node in the basic pattern, and the sum is taken over all such patterns which are glued up. By the symmetry of $M_{1}, M_{3}$, and $M_{5}$, we have $n(i)=4$ for $M_{1}$ and $M_{3}$, and $n(i)=6$ for $M_{5}$. For $M_{2}, n(i)$ takes values $3,4,5$, see figure 3 . For $i \in V_{k}^{(l)}, l=2,3, \ldots, k-1$, we have $n(i)=\tilde{n}(i)+\nu(i)$, where $\tilde{n}(i)$ is non-random and has to be calculated as just described. The summand $\nu(i)$ is the number of decorating bonds attached to $i$. To simplify our consideration, let us stick to the case of the graph generated by $M_{1}$. Then for $l=1, \ldots, k-1$ and $i \in V_{k}^{(l)}$, we have $\tilde{n}(i)=4$ and $\nu(i)$ takes values $\nu=0,1,2, \ldots, 4(l-1)$, with probability

$$
\operatorname{Prob}(\nu(i)=\nu)=\left(\begin{array}{c}
4(l-1) \\
\nu
\end{array}\right) p^{\nu}(1-p)^{4(l-1)-\nu} .
$$

For $i \in V_{k}^{(k)}, \nu(i)$ takes values $0,1, \ldots, 2(k-1)$. Therefore, the maximum node degree which can occur in $V_{k}, k \geqslant 2$, is

$$
\max _{i \in V_{k}} n(i)=4 k-4 .
$$

As is usual in the theory of real world networks, which are in fact non-random, the randomness manifests itself as the random choice of a node. If we apply this principle here, then (13) can be considered as the conditional probability distribution, conditioned by the event that the node $i$ 
has been picked from the set $V_{k}^{(l)}$. The probability of the latter event is taken to be proportional to the number of its elements, that is,

$$
\begin{aligned}
& \operatorname{Prob}\left(i \in V_{k}^{(l)}\right)=\frac{\left|V_{k}^{(l)}\right|}{\left|V_{k}\right|}=\frac{q-1}{1+q^{1-k}} q^{-l}=\frac{2}{1+3^{1-k}} 3^{-l}, \quad l \leqslant k-1, \\
& \operatorname{Prob}\left(i \in V_{k}^{(k)}\right)=\frac{2 q}{q^{k}+q}=\frac{2}{3^{k-1}+1} .
\end{aligned}
$$

If we now take the expectation of $n(i)$ with respect to this distribution, that is,

$$
\begin{aligned}
\left\langle n_{k}\right\rangle & =\sum_{l=1}^{k-1} \sum_{\nu=0}^{4(l-1)}(4+\nu) \frac{2 \cdot 3^{-l}}{1+3^{1-k}} \cdot\left(\begin{array}{c}
4(l-1) \\
\nu
\end{array}\right) p^{\nu}(1-p)^{4(l-1)-\nu} \\
& +\sum_{\nu=0}^{2(k-1)}(2+\nu) \frac{2}{3^{k-1}+1} \cdot\left(\begin{array}{c}
2(k-1) \\
\nu
\end{array}\right) p^{\nu}(1-p)^{2(k-1)-\nu}
\end{aligned}
$$

we readily obtain $\left\langle n_{k}\right\rangle=4+2 p-4(1+p) /\left(3^{k-1}+1\right)$, which is in full agreement with the one given in (11) or in table 11. In order to figure out the limit $k \rightarrow+\infty$ of the distribution given by (13) and (15) we calculate its characteristic function, c.f. (16),

$$
\begin{aligned}
\varphi_{k}(t) & =\sum_{l=1}^{k-1} \sum_{\nu=0}^{4(l-1)} \exp [\mathrm{i} t(4+\nu)] \frac{2 \cdot 3^{-l}}{1+3^{1-k}} \cdot\left(\begin{array}{c}
4(l-1) \\
\nu
\end{array}\right) p^{\nu}(1-p)^{4(l-1)-\nu} \\
& +\sum_{\nu=0}^{2(k-1)} \exp [\mathrm{i} t(2+\nu)] \frac{2}{3^{k-1}+1} \cdot\left(\begin{array}{c}
2(k-1) \\
\nu
\end{array}\right) p^{\nu}(1-p)^{2(l-1)-\nu} \\
& =\frac{2 e^{4 \mathrm{i} t}}{1+3^{1-k}} \cdot \frac{1-3^{1-k}\left(e^{\mathrm{i} t} p+1-p\right)^{4}}{3-\left(e^{\mathrm{i} t} p+1-p\right)^{4}} \\
& +\frac{2 e^{2 \mathrm{i} t}}{3^{k-1}+1}\left(e^{\mathrm{i} t} p+1-p\right)^{2}, \quad \mathrm{i}=\sqrt{-1}
\end{aligned}
$$

Then the limiting characteristic function is

$$
\varphi(t)=\frac{2 e^{4 i t}}{3-\left(e^{\mathrm{i} t} p+1-p\right)^{4}},
$$

which can be continued to a meromorphic function analytic in some complex neighborhood of the point $t=0$. This means that the limiting node degree distribution has all moments and hence cannot be of scale-free typ 3 . This also agrees with the heuristic rule, see equation (3.13) on page 188 in [20], that for scale-free networks

$$
\max _{i \in V_{k}} n(i) \sim\left|V_{k}\right|^{1 /(\alpha-1)}, \quad \alpha>1
$$

whereas in our case we have (14) and $\left|V_{k}\right|=\left(3^{k}+3\right) / 2$. In a similar way, one can show that all our graphs are not scale-free. Another observation on this item can be made by comparing the function (18) with the characteristic function of the Poisson distribution (2) which has the form

$$
\varphi_{\text {Poisson }}(t)=\exp \left[c\left(e^{\mathrm{i} t}-1\right)\right],
$$

and hence can be continued to a function analytic on the whole complex plane. Therefore, the distribution corresponding to (18) with $p>0$ is intermediate as compared to the Poisson and scale-free distributions. For $p=0$, the function (18) is also entire.

\footnotetext{
${ }^{3}$ For scale-free graphs, the node degree distribution is $P(k)=C k^{-\gamma}, k \geqslant 1, \gamma>1$; hence, $\sum_{k=1}^{\infty} k^{m} P(k)$ diverges for all $m \geqslant \gamma-1$.
} 


\subsection{Amenability, clustering, and small world properties}

The next property of our graphs which we are going to address is amenability. To introduce it we need one more notion. Let $G=(V, E)$ be a countable graph with node set $V$ and bond set $E$. For a finite $\Delta \subset V$, by $\partial \Delta$ we denote the set of nodes which are not in $\Delta$ but have neighbors in $\Delta$. This set is the outer boundary of $\Delta$, whereas the elements of $\Delta$ which are neighbors to the elements of $\partial \Delta$ constitute the inner boundary of $\Delta$. As usual, by $|\Delta|$ and $|\partial \Delta|$ we denote the number of nodes in these sets. The graph $G$ is said to be amenable if there exists a sequence of finite node sets $\left\{\Delta_{k}\right\}_{k \in \mathbb{N}}$, such that

$$
\lim _{k \rightarrow+\infty} \frac{\left|\partial \Delta_{k}\right|}{\left|\Delta_{k}\right|}=0
$$

If such a limit is positive for any sequence $\left\{\Delta_{k}\right\}_{k \in \mathbb{N}}$, the graph is called nonamenabile. The lattices $\mathbb{Z}^{d}, d \geqslant 1$, are amenable graphs and for such sets one can take the cubes

$$
\Delta_{k}=\left\{i=\left(i_{1}, \ldots i_{d}\right) \in \mathbb{Z}^{d}:\left|i_{j}\right| \leqslant N_{k}, j=1, \ldots, d\right\},
$$

such that $N_{k+1}>N_{k}$ and $N_{k} \rightarrow+\infty$. In this case, $\left|\Delta_{k}\right| \sim N_{k}^{d}$ and $\left|\partial \Delta_{k}\right| \sim N_{k}^{d-1}$ and hence (20) holds. Sometimes, sequences for which (20) holds are called Van Howe sequences, see e.g. [21]. Cayley trees, except for $\mathbb{Z}$, are nonamenable. Let us turn now to our graphs. Due to their hierarchical structure, it is convenient to check (20) for the sequence of node sets of $\Lambda_{k}$, that is for $\left\{V_{k}\right\}_{k \in \mathbb{N}}$. By the construction of $\Lambda_{k}$, the inner boundary of each $V_{k}$ is exactly the set of all its external nodes, the number of which is equal to the number of nodes in the corresponding motif, i.e. it is $q$. By construction, one of them becomes an external node of $\Lambda_{k+1}$, and the remaining $q-1$ ones become inner nodes of $\Lambda_{k+1}$. For all the motifs $M_{j}, j=1, \ldots, 5$, we have the degree of any node therein being at most 3 , see figure 1 Then for all our graphs,

$$
\max _{i \in V_{k}} n(i) \leqslant 6 k,
$$

c.f. (14). At the same time, $\left|V_{k}\right| \sim q^{k}, q=3,4$, see (9), which immediately yields that all our graphs are amenable.

Now we study the clustering in our graphs. For non-random graphs, the clustering coefficient is defined as follows. For a given node $i \in V$ of degree $n(i)$, let $N(i)$ be the number of bonds linking its neighbors, which is the number of triangles with vertex $i$. Clearly, $N(i) \leqslant n(i)[n(i)-1] / 2$ and the maximum value of this parameter is attained for complete graphs where each node is a neighbor to any other one. Thus, the quantity

$$
Q(i):=\frac{2 N(i)}{n(i)[n(i)-1]}
$$

characterizes clustering at node $i$. Then we define the clustering of our graphs as

$$
Q=\lim _{k \rightarrow+\infty} \frac{1}{\left|V_{k}\right|} \sum_{i \in V_{k}} Q(i) \text {. }
$$

Note that for many graphs, e.g., for trees or bipartite graphs, one has $Q(i)=0$ for any node $i$, see also [22, 23]. For random graphs, the degree $n(i)$, as well as the parameter $N(i)$, are random. Since in this case the calculation of $Q$ is much more involved, we postpone it to the future. Here we only compare the values of $Q$ obtained for the bare graphs with those for fully decorated ones.

For the graph based on $M_{1}$ and a node $i \in V_{k}^{(l)}, l=1, \ldots, k-1$, we have: (a) $n(i)=4, N(i)=3$ for $l=1$, and $N(i)=2$ for $l \geqslant 2$; (b) $n(i)=4 l, N(i)=4 l$. Here (a) and (b) correspond to a bare graph and to a fully decorated graph, respectively. These numbers follow directly from the construction of the graphs. The number of elements in each $V_{k}^{(l)}$ is given in (12), which allows one to compute

$$
\text { (a) } Q=\lim _{k \rightarrow+\infty}\left(\frac{1}{3}+\frac{\left|V_{k}^{(1)}\right|}{6\left|V_{k}\right|}+\frac{2}{\left|V_{k}\right|}\right)=\frac{4}{9}=0.4444 \ldots \text {, }
$$




$$
\text { (b) } Q=2 \cdot 3^{-1 / 4} \arctan 3^{-1 / 4}-\frac{1}{3^{1 / 4}} \ln \frac{3^{1 / 4}+1}{3^{1 / 4}-1} \approx 0.525897 \text {. }
$$

For the bare graph based on $M_{3}$, we have $N(i)=0$ for all nodes; hence, $Q=0$ in this case. For the fully decorated graph based on $M_{3}$, we observe that two nodes of $V_{2}^{(1)}$ have neighbors only in $V_{2}^{(1)}$, and the remaining four nodes have two neighbors in $V_{2}^{(1)}$ and two - in $V_{2}^{(2)}$. Since for the nodes $i \in V_{2}^{(1)}$, we have $N(i)=0$, like in the case of the bare graph, we should consider these two groups separately. Thus, we split each $V_{k}^{(l)}, l=1, \ldots, k-1$, into $V_{k, 0}^{(l)}$ and $V_{k, 1}^{(l)}$, where the first set consists of the nodes which have neighbors only in $V_{k, 0}^{(l)}$. The elements of $V_{k, 1}^{(l)}$ have also neighbors in $V_{k}^{\left(l^{\prime}\right)}$ with $l^{\prime}>l$. The number of nodes in these sets are:

$$
\left|V_{k, 0}^{(l)}\right|=\frac{1}{2} 4^{k-l}, \quad\left|V_{k, 1}^{(l)}\right|=4^{k-l} .
$$

For all $i \in V_{k}^{(l)}, l=1, \ldots, k-1$, we have $n(i)=4 l$. At the same time, $N(i)=4(l-1)$ for $i \in V_{k, 0}^{(l)}$, and $N(i)=1+4(l-1)$ for $i \in V_{k, 1}^{(l)}$. Putting all these numbers together we obtain

$$
Q=\frac{3}{2} \sum_{l=2}^{\infty} \frac{(l-1) 4^{-(l-1)}}{l(4 l-1)}+\sum_{l=1}^{\infty} \frac{4^{-l}}{l(4 l-1)} \approx 0.1223 .
$$

For the bare graph based on $M_{5}$ one can obtain for $i \in V_{k}^{(l)}, l=1,2, \ldots, k-1: n(i)=6, N(i)=8$ for $l=1$, and $n(i)=6, N(i)=6$ for $l \geqslant 2$. For the fully decorated graph based on $M_{5}$ we have for all internal nodes $n(i)=6 l$, and $N(i)=9$ for $l=1$, and $N(i)=12 l+7$ for $l \geqslant 2$. Hence, we obtain

$$
Q=\lim _{k \rightarrow+\infty}\left(\frac{2}{5}+\frac{2\left|V_{k}^{(1)}\right|}{15\left|V_{k}\right|}+\frac{12}{5\left|V_{k}\right|}\right)=0.5
$$

$$
Q \approx 0.554145
$$

There exists one more property of real world networks, which Erdős-Rényi type graph does not share, see e.g. [20, 24]. It is the so-called small-world property, illustrated by Pal Erdős himself in the following way. All authors of mathematical papers are given the Erdös index which for his coauthors is equal to one. For their coauthors who are not Erdös' coauthors, this index is equal to 2, and so on. Everyone whose papers are indexed by the Mathematical Reviews can check his own index at the web-site http://www.ams.org/mathscinet/. It turns out that for many authors it ranges from 2 to 6 (for the second named author of this paper it is 4 ). To formulate the small-world property one needs the following notion. A path in the graph is a sequence of nodes such that every two consecutive elements of this sequence are neighbors to each other. The length of the path is the number of such consecutive pairs, which is equal to the number of bonds one passes on the way from the origin to the terminus. If every two nodes can be connected by a path, the graph is said to be connected. For the given two nodes, $i$ and $j$, the length of the shortest path which connects them is said to be the distance $\rho(i, j)$ between these nodes. Informally speaking, a graph $G=(V, E)$ has the small-world property (or is a small-world graph) if every two nodes $i, j \in V$ are "not too far" from each other. More precisely this property is formulated as follows. An infinite graph $G$ has a small-world property if there exists a sequence of its connected finite subgraphs $\left\{G_{k}\right\}_{k \in \mathbb{N}}$ with the following property. Let $\operatorname{diam}\left(G_{k}\right)=\max _{i, j \in V_{k}} \rho(i, j)$ be the diameter of $G_{k}$, $k \in \mathbb{N}$, and $\left\langle n_{k}\right\rangle$ be the average value of the node degree in $G_{k}$, that is, $\left\langle n_{k}\right\rangle=2\left|E_{k}\right| /\left|V_{k}\right|$. Then the sequence $\left\{G_{k}\right\}_{k \in \mathbb{N}}$, and hence the graph $G$, are said to have the small-world property if there exists a positive constant $C$ such that for all $k \in \mathbb{N}$,

$$
\operatorname{diam}\left(G_{k}\right) \leqslant C \log _{\left\langle n_{k}\right\rangle}\left|V_{k}\right| .
$$

This means that in such graphs, the distances between the nodes scale at most logarithmically with the size of the graph. For our graphs, the results on this item are given in the last three rows 
of table 1. The diameters of $\Lambda_{k}$ presented therein were calculated in a routine way for the cases where the graphs are bare $(p=0)$ or are fully decorated $(p=1)$. In the former case, neither of our graphs has the small-world property. However, this property holds for all fully decorated graphs.

Table 1. The structural characteristics of the families of hierarchical graphs.

\begin{tabular}{|c|c|c|c|c|c|}
\hline motif & $M_{1}$ & $M_{2}$ & $M_{3}$ & $M_{4}$ & $M_{5}$ \\
\hline$\left|V_{k}\right|$ & $\frac{3}{2}\left(3^{k-1}+1\right)$ & $2\left(4^{k-1}+1\right)$ & $2\left(4^{k-1}+1\right)$ & $2\left(4^{k-1}+1\right)$ & $2\left(4^{k-1}+1\right)$ \\
\hline$\left|E_{k}\right|$ & $3^{k}$ & $4^{k}$ & $4^{k}$ & $5 \cdot 4^{k-1}$ & $6 \cdot 4^{k-1}$ \\
& $\frac{3}{2}\left(3^{k-1}-1\right) p$ & $\frac{4}{3}\left(4^{k-1}-1\right) p$ & $\frac{4}{3}\left(4^{k-1}-1\right) p$ & $\frac{5}{3}\left(4^{k-1}-1\right) p$ & $2\left(4^{k-1}-1\right) p$ \\
\hline$\left\langle n_{k}\right\rangle$ & $4+2 p$ & $4+\frac{4}{3} p$ & $4+\frac{4}{3} p$ & $5+\frac{5}{3} p$ & $6+2 p$ \\
& $-4 \frac{1+p}{3^{k-1}+1}$ & $-\frac{4}{3} \frac{3+2 p}{4^{k-1}+1}$ & $-\frac{4}{3} \frac{3+2 p}{4^{k-1}+1}$ & $-\frac{5}{3} \frac{3+2 p}{4^{k-1}+1}$ & $-2 \frac{3+2 p}{4^{k-1}+1}$ \\
\hline $\operatorname{diam}\left(\Lambda_{k}\right), p=0$ & $2^{k-1}$ & $2^{k}$ & $2^{k}$ & $2^{k}$ & $2^{k-1}$ \\
\hline $\operatorname{diam}\left(\Lambda_{k}\right), p=1$ & $k$ & $k+1$ & $2(k-1)$ & $k$ & $k$ \\
\hline$C, p=1$ & $\left(\log _{6} 3\right)^{-1}$ & $\left(\log _{6} 4\right)^{-1}$ & $2\left(\log _{6} 4\right)^{-1}$ & $\left(\log _{7} 4\right)^{-1}$ & $\left(\log _{8} 4\right)^{-1}$ \\
\hline
\end{tabular}

In table 1, we collected the structural characteristics of our graphs based on the motifs $M_{1}, \ldots$, $M_{5}$ given in figure 1, Its first three rows are obtained from the formulas (9) and (11).

\section{Graph structure and the Ising model properties}

As was mentioned above, there exists a profound connection between the properties of Gibbs random fields of models based on graphs and the structural properties of these graph 4 . For the hierarchical lattices, the notion of the Gibbs random field of the Ising model was introduced in [14, 15]. In the physical terminology, each (pure) Gibbs random field corresponds to a state of thermal equilibrium of the model, see 25] for more details. Accordingly, the existence of multiple Gibbs random fields corresponds to the existence of multiple equilibrium states and hence of phase transitions. If there is no interaction between the spins, the Gibbs random field is unique. However, if the interaction is strong enough and if it is effectively propagated by the underlying graph (high enough "connectivity"), the Gibbs fields can be multiple. The condition of high connectivity is essential, which can be seen from the fact that the Ising model on the one-dimensional lattice $\mathbb{Z}$, considered as a graph with the natural adjacency relation, bears only one Gibbs field and hence no phase transitions can occur in this case - no matter how strong the interaction is. With regard to these arguments, we can divide all graphs into three groups according to the following property of the Ising model defined on these graphs:

(a) There exists only one Gibbs random field for all interactions.

(b) There exists only one Gibbs random field for weak interactions, and multiple Gibbs random fields for strong interactions.

(c) There exist multiple Gibbs random fields for all nonzero interactions.

${ }^{4}$ Of course, we are speaking now about countably infinite graphs. 
As was mentioned above, the lattice $\mathbb{Z}$ belongs to the first group. The lattices $\mathbb{Z}^{d}$ with $d \geqslant 2$, as well as all Cayley trees 5 belong to the second group. An example of the graph belonging to the third group can be found in section 4 of [26]. Clearly, the above classification should be made more precise for random graphs, since in this case the existence of a Gibbs random field is a random event. We return to this issue below.

The Ising model defined on the graphs from the second group may have a critical point, which separates the regime of uniqueness (weak interactions) from the regime of non-uniqueness (strong interactions). At their critical point, the Gibbs random fields have "unusual" properties, which can be detected without explicit construction of these fields. One of such properties is the socalled self-similarity, which manifests itself in the appearance of unstable fixed points of some (renormalization) transformations. It turns out that the hierarchical graphs are quite suitable for such a study - that it why they appear in this context. Thus, one can have the following criterion: if the Ising model has a critical point, the graph belongs to the second group. If not, then the graph belongs either to the first or to the third group. The latter cases can be distinguished by an additional study.

In contrast to the hierarchical lattices introduced and studied in [16], our bare graphs belong to the first group. This can be seen from the analysis which we present below - a direct proof of such a statement will be done in our forthcoming publication. Thus, the interaction along the decorating bonds plays the main role in the possible appearance of phase transitions in the Ising model defined on our graphs. In view of this fact, we will assume that the solid bonds bear the interaction $K$, whereas the dotted bonds bear the interaction $L$. As the bonds of the latter kind appear at random, we can think of the corresponding model as of the one defined on the fully decorated graph with random interactions along the decorating bonds. Thus, in this model, the interaction along each bond $\langle i, j\rangle$ is a random variable, denoted by $L_{i j}^{\omega}$, which takes values 0 and some nonzero $L \in \mathbb{R}$ with probabilities $1-p$ and $p$, respectively. For different bonds, these random variables are independent.

For a given $k \in \mathbb{N}$, the Ising model on the fully decorated graph $\Lambda_{k}$ is defined in the usual way by assigning spin variables $\sigma_{i}= \pm 1$ to the nodes $i \in V_{k}$ and by setting the Hamiltonian

$$
-\beta \mathcal{H}_{k}=h \sum_{i \in V_{k}} \sigma_{i}+K \sum_{\langle i, j\rangle \in E_{k}^{\prime}} \sigma_{i} \sigma_{j}+\sum_{\langle i, j\rangle \in E_{k}^{\prime \prime}} L_{i j}^{\omega} \sigma_{i} \sigma_{j}, \quad h, K \in \mathbb{R}, \quad k \in \mathbb{N},
$$

where $h$ is an external field. In accordance with the above arguments, the third summand corresponds to the interaction along decorating bonds. We have included the first term in view of the following arguments. As is known, the Ising model on the lattices $\mathbb{Z}^{d}, d \geqslant 2$, exhibits phase transitions only if $h=0$. This is also the case if the graph is amenable and quasi-transitive. For nonamenable graphs, this model may have a phase transition for nonzero $h$, see [27].

By the construction of our graphs, each $\Lambda_{k}$ has $q$ external nodes, $i_{1}, \ldots, i_{q}$, and the corresponding number of internal nodes, see table 1. Such external nodes can be considered as a boundary of $\Lambda_{k}$. Correspondingly, we call the spin variables external or boundary (respectively, internal) spins if they are assigned to external (respectively, internal) nodes. For a fixed configuration of the external spins $\sigma_{i_{1}}, \ldots, \sigma_{i_{q}}$, we consider

$$
Z_{k}^{\omega}\left(\sigma_{i_{1}}, \ldots, \sigma_{i_{q}}\right)=\sum_{\text {internal spins of } \Lambda_{k}} \exp \left(-\beta \mathcal{H}_{k}\right) .
$$

This is the conditional partition function of the spin system in $\Lambda_{k}$, conditioned by the fixed configuration of spins on the boundary of $\Lambda_{k}$. Of course, it depends on the graph, i.e. on the motif used in its construction. According to the main principle of the theory of Gibbs random fields applied in our context, see [25], the fact that such a field is unique is ensured by the conditional partition function asymptotic independence, as $k \rightarrow+\infty$, of the boundary spins. For our graphs, $Z_{k}^{\omega}$ are random and hence also depend on $\omega$. In this situation, one can apply the following two approaches: the above mentioned independence holds (i) for almost all $\omega$; (ii) in average. The latter

\footnotetext{
${ }^{5}$ Except for $\mathbb{Z}$, see page 247 in 25$]$.
} 
corresponds to the fact that the expected values of $Z_{k}^{\omega}$, which we denote by $\left\langle Z_{k}^{\omega}\right\rangle$, are independent of the boundary spins. These two approaches are called quenched and annealed disorders, respectively, see [28], page 99. We will work in the annealed approach. It can be shown that the summands in (29) are positive and separated from zero. Since the number of these summands increases to $+\infty$ as $k \rightarrow+\infty$, we have $\left\langle Z_{k}^{\omega}\right\rangle \rightarrow+\infty$. Therefore, a weaker form of the uniqueness condition can be

$$
\lim _{k \rightarrow+\infty}\left\langle Z_{k}^{\omega}\left(\sigma_{i_{1}}, \ldots, \sigma_{i_{q}}\right)\right\rangle /\left\langle Z_{k}^{\omega}\left(\sigma_{i_{1}}^{\prime}, \ldots, \sigma_{i_{q}}^{\prime}\right)\right\rangle=c,
$$

which holds for any two configurations of the boundary spins and for some $c>0$, which depends on these configurations. The convergence in (30) should be stable with respect to small changes of $h$ and $K$, i.e. of the starting element $Z_{1}$. In this case, the annealed limiting free energy per spin is independent of the boundary configuration. Correspondingly, a necessary condition for the latter quantity to be dependent on the boundary spins is that

$$
\lim _{k \rightarrow+\infty}\left\langle Z_{k}^{\omega}\left(\sigma_{i_{1}}, \ldots, \sigma_{i_{q}}\right)\right\rangle /\left\langle Z_{k}^{\omega}\left(\sigma_{i_{1}}^{\prime}, \ldots, \sigma_{i_{q}}^{\prime}\right)\right\rangle=+\infty
$$

for some $\sigma_{i_{1}}, \ldots, \sigma_{i_{q}}$ and $\sigma_{i_{1}}^{\prime}, \ldots, \sigma_{i_{q}}^{\prime}$. We say that the Ising model defined on our graph has a critical point, if there exists $c^{\prime}>0$, distinct from $c$ in (30), such that for $K=K_{*}$ and $h=h_{*}$,

$$
\lim _{k \rightarrow+\infty}\left\langle Z_{k}^{\omega}\left(\sigma_{i_{1}}, \ldots, \sigma_{i_{q}}\right)\right\rangle /\left\langle Z_{k}^{\omega}\left(\sigma_{i_{1}}^{\prime}, \ldots, \sigma_{i_{q}}^{\prime}\right)\right\rangle=c^{\prime}
$$

Here $K_{*}$ and $h_{*}$ are certain values of the interaction intensity and the external field. The convergence in (32) should be such that for arbitrarily small deviations from the point $\left(K_{*}, h_{*}\right)$ one has either (30) or (31) instead of (32). Note that we do not exclude that $h_{*} \neq 0$ since our graphs are not quasi-transitive and hence the result of [27] may not be applicable in this case.

\section{Critical points of the Ising model on $M_{1}$ based graph}

For $k=1$, we have only basic bonds and no inner nodes. Thus,

$$
Z_{1}(a, b, c)=\exp \left[-\beta \mathcal{H}_{1}(a, b, c)\right]=\exp [K(a b+a c+b c)+h(a+b+c)],
$$

where we use the same notations for the nodes $a, b, c$, see the left-hand graph in figure 2, as well as for the corresponding spin variable, that is, for short we write $a=\sigma_{a}, b=\sigma_{b}$, ect. For $k=2$, we have, see the middle graph in figure 2 .

$$
-\beta \mathcal{H}_{2}(a, b, c, \alpha, \beta, \gamma)=-\beta \mathcal{H}_{1}(a, \gamma, \beta)-\beta \mathcal{H}_{1}(\gamma, b, \alpha)-\beta \mathcal{H}_{1}(\beta, \alpha, c)+L_{a b}^{\omega} a b+L_{b c}^{\omega} b c+L_{a c}^{\omega} a c .
$$

Thus, to obtain $Z_{2}(a, b, c)$ we have to sum out the internal spins, see (29). That is,

$$
Z_{2}^{\omega}(a, b, c)=\exp \left[L_{a b}^{\omega} a b+L_{a c}^{\omega} a c+L_{b c}^{\omega} b c\right] \sum_{\alpha, \beta, \gamma} Z_{1}(a, \gamma, \beta) Z_{1}(\gamma, b, \alpha) Z_{1}(\beta, \alpha, c),
$$

where the sum is taken over $\alpha, \beta, \gamma= \pm 1$. According to the hierarchical structure of the underlying graph, for all $k \geqslant 2$ the graph $\Lambda_{k}$ is obtained from the subgraphs $\Lambda_{k-1}$ exactly in the same way. This yields the following recurrence for the partition functions (29)

$$
Z_{k}^{\omega}(a, b, c)=R_{k}^{\omega}(a, b, c) \sum_{\alpha, \beta, \gamma} Z_{k-1}(a, \gamma, \beta) Z_{k-1}(\gamma, b, \alpha) Z_{k-1}(\beta, \alpha, c)
$$

where

$$
R_{k}^{\omega}(a, b, c)=\exp \left[L_{a b}^{\omega} a b+L_{a c}^{\omega} a c+L_{b c}^{\omega} b c\right],
$$

and $Z_{1}(a, b, c)$ is given in (33). From the independence of $L_{i j}^{\omega}$ for different bonds, it follows that the random variables $Z_{k-1}^{\omega}$ on the right-hand side of (34) are independent, which allows us to compute the expectations and obtain

$$
\left\langle Z_{k}^{\omega}(a, b, c)\right\rangle=\left\langle R_{k}^{\omega}(a, b, c)\right\rangle \sum_{\alpha, \beta, \gamma}\left\langle Z_{k-1}^{\omega}(a, \beta, \gamma)\right\rangle\left\langle Z_{k-1}^{\omega}(\alpha, b, \gamma)\right\rangle\left\langle Z_{k-1}^{\omega}(\alpha, \beta, c)\right\rangle .
$$


We recall that the random variables $L_{i j}^{\omega}$ take the values $L \neq 0$ and 0 with probabilities $p$ and $1-p$, respectively. In view of the model symmetry, c.f. (33), the system (36) involves the following variables

$$
\begin{array}{ll}
A_{k}=\left\langle Z_{k}^{\omega}(1,1,1)\right\rangle, & B_{k}=\left\langle Z_{k}^{\omega}(1,1,-1)\right\rangle \\
C_{k}=\left\langle Z_{k}^{\omega}(-1,-1,1)\right\rangle, & D_{k}=\left\langle Z_{k}^{\omega}(-1,-1,-1)\right\rangle
\end{array}
$$

which have the initial values

$$
A_{1}=e^{3(K+h)}, \quad B_{1}=e^{-K+h}, \quad C_{1}=e^{-K-h}, \quad D_{1}=e^{3(K-h)} .
$$

By direct calculations,

$$
\begin{aligned}
\left\langle R_{k}^{\omega}(1,1,1)\right\rangle & =(p \exp (L)+1-p)^{3} \\
\left\langle R_{k}^{\omega}(-1,1,1)\right\rangle & =(p \exp (L)+1-p)(p \exp (-L)+1-p)^{2} .
\end{aligned}
$$

Thereafter, the recursion (36) can be written in the form

$$
\begin{aligned}
x_{k+1} & =t \frac{P_{x}\left(x_{k}, y_{k}\right)}{Q\left(x_{k}, y_{k}, z_{k}\right)}, \\
y_{k+1} & =\frac{P_{y}\left(x_{k}, y_{k}, z_{k}\right)}{Q\left(x_{k}, y_{k}, z_{k}\right)}, \\
z_{k+1} & =t \frac{P_{z}\left(y_{k}, z_{k}\right)}{Q\left(x_{k}, y_{k}, z_{k}\right)},
\end{aligned}
$$

with the initial conditions

$$
x_{1}=e^{4(K+h)}, \quad y_{1}=e^{2 h}, \quad z_{1}=e^{4 K-2 h},
$$

where we have used the notations

$$
x_{k}=A_{k} / C_{k}, \quad y_{k}=B_{k} / C_{k}, \quad z_{k}=D_{k} / C_{k}, \quad k \in \mathbb{N},
$$

and

$$
t=\left(\frac{p e^{L}+1-p}{p e^{-L}+1-p}\right)^{2} .
$$

The polynomials in (40) have the following form

$$
\begin{aligned}
P_{x}(x, y) & =x^{3}+3 x y^{2}+3 y^{2}+1, \\
P_{y}(x, y, z) & =x^{2} y+y^{3}+2 x y+y^{2} z+2 y+z, \\
P_{z}(y, z) & =y^{3}+3 y+3 z+z^{3}, \\
Q(x, y, z) & =x y^{2}+x+2 y^{2}+2 y z+z^{2}+1 .
\end{aligned}
$$

For every $t>0$, the mapping $\left(x_{k}, y_{k}, z_{k}\right) \mapsto\left(x_{k+1}, y_{k+1}, z_{k+1}\right)=T_{t}\left(x_{k}, y_{k}, z_{k}\right)$, defined in (40) and (44), maps the first octant of $\mathbb{R}^{3}$ into itself. The starting point $\left(x_{1}, y_{1}, z_{1}\right)$ lies on the surface $\mathcal{S}$ defined by the equation, c.f. (41),

$$
x=y^{3} z,
$$

which, however, is not preserved by this mapping, which can be checked directly. Let $\mathcal{T}_{t}$ be the invariant set of the map $T_{t}$, that is, the set of points $(x, y, z)$ such that $T_{t}(x, y, z)=(x, y, z)$. Suppose that the intersection of $\mathcal{T}_{t}$ with $\mathcal{S}$ is non-void. If $\left(x_{*}, y_{*}, z_{*}\right)$ belongs to this intersection 


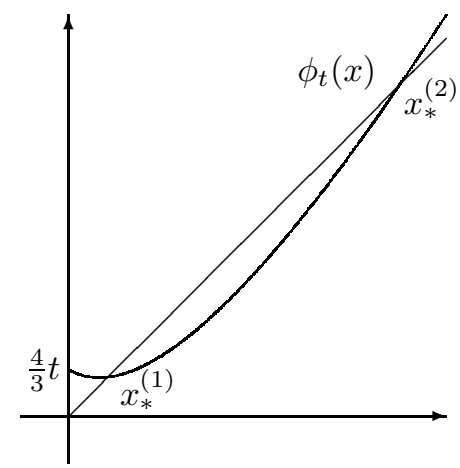

Figure 5. Graphical solution of (48).

and $\left(x_{1}, y_{1}, z_{1}\right)=\left(x_{*}, y_{*}, z_{*}\right)$, then $\left(x_{k}, y_{k}, z_{k}\right)=\left(x_{*}, y_{*}, z_{*}\right)$ for all $k \in \mathbb{N}$. Let us find such points. From the second equation in (40) for $x=y^{3} z$ we get

$$
y Q\left(y^{3} z, y, z\right)=P_{y}\left(y^{3} z, y, z\right),
$$

which can be transformed into the following

$$
y^{3}\left(1+y^{3} z\right)(1-y z)=(y+z)(1-y z) .
$$

A solution of the latter equation is $z=1 / y$, thus $x=y^{2}$, which being used in the first equation in (40) yields $\left(1+y^{2}\right)^{2}=t\left(1+y^{2}\right)^{2}$ and hence $t=1$. For $p>0$, the latter implies $L=0$, and also $K=0$, see (41). This fixed point corresponds to a noninteracting system of spins in an external field and thereby has nothing to do with critical points. For $z \neq 1 / y$, from (46) we get

$$
z\left(1-y^{2}\right)\left(y^{4}+y^{2}+1\right)=-y\left(1-y^{2}\right),
$$

which for positive $y$ and $z$ has a unique solution $y=1$, and hence $x=z$. We insert this into the first equation in (40) and obtain

$$
x=t \frac{x^{3}+3 x+4}{x^{2}+4 x+3}=t \frac{x^{2}-x+4}{x+3}:=\phi_{t}(x) .
$$

For $t=1$, the only solution is $x=1$, which corresponds to $L=K=0$. The choice $t=1$ also corresponds to a bare graph $(p=0)$; hence, the bare graph based on $M_{1}$ has no critical points and no phase transitions. As we have already mentioned above, in a separate work we prove that the Gibbs states of the Ising model based on this graph are always unique. For $t<1$, that is, for $L<0$, (48) has only one positive solution

$$
x_{*}=\frac{-3-t+\sqrt{9+22 t-15 t^{2}}}{2(1-t)}<1,
$$

which corresponds to $K_{*}<0$. It is stable and $\left(x_{k}, y_{k}, z_{k}\right) \rightarrow\left(x_{*}, 1, x_{*}\right)$ as $k \rightarrow+\infty$, for any $\left(x_{1}, y_{1}, z_{1}\right) \in \mathcal{S}$. Thus, for $L<0$, the Ising model has no critical points and no phase transitions, even for the fully decorated graph. This can be caused by a frustration, which takes place in an antiferromagnetic Ising model on triangles. For $t>1$, figure 5 presents the graphical solutions of (48). They have the following form

$$
x_{*}^{(1)}=\frac{3+t-\sqrt{9+22 t-15 t^{2}}}{2(t-1)}, \quad x_{*}^{(2)}=\frac{3+t+\sqrt{9+22 t-15 t^{2}}}{2(t-1)} .
$$

By direct calculations, we get $\phi_{t}^{\prime}\left(x_{*}^{(1)}\right)<1$ and $\phi_{t}^{\prime}\left(x_{*}^{(2)}\right)>1$, see figure 5 . Hence, $x_{*}^{(1)}$ is stable, whereas $x_{*}^{(2)}$ is unstable. Note also that $x_{*}^{(1)} \rightarrow 1$ and $x_{*}^{(2)} \rightarrow \infty$ as $t \rightarrow 1$. The solutions (50) exist and are considered to be distinct, provided $t \in(1,9 / 5)$. This yields the condition

$$
p<\psi(L):=\frac{3-\sqrt{5}}{\sqrt{5} \exp (L)-3 \exp (-L)+3-\sqrt{5}} .
$$


Since $\psi(L)$ is a decreasing function, the equation $\psi(L)=1$ has only one solution

$$
L_{*}=\frac{1}{4} \ln \frac{9}{5} \text {. }
$$

Then, for $L \leqslant L_{*}$, the critical point exists for all $p \in(0,1]$. For such $L$ and $p$, let $K_{*}(L, p)$ be the solution of the equation

$$
\exp (4 K)=x_{*}^{(2)}
$$

Then

$$
\lim _{k \rightarrow+\infty} x_{k}= \begin{cases}x_{*}^{(1)} & \text { if } x_{1}<\exp \left[4 K_{*}(L, p)\right] \\ x_{*}^{(2)} & \text { if } x_{1}=\exp \left[4 K_{*}(L, p)\right] \\ +\infty & \text { if } x_{1}>\exp \left[4 K_{*}(L, p)\right]\end{cases}
$$

The conditions in (54) can be formulated directly for $K$, e.g. $x_{k} \rightarrow x_{*}^{(1)}$ if $K<K_{*}(L, p)$. Thus, $x_{*}^{(1)}$ is the so-called high-temperature fixed point. For $L>L_{*}$, we have $\psi(L)<1$. Hence, the condition (51) is not satisfied for $p \in(\psi(L), 1]$, which also includes the fully decorated graph with $p=1$. For such $L$ and $p$, the whole graph of $\phi_{t}$ lies above the line $\phi_{t}(x)=x$, which means that $x_{k} \rightarrow+\infty$ for all initial $x_{1} \geqslant 0$. Thus, for $L>L_{*}$ and $p \in\left(\psi\left(L_{*}\right), 1\right]$, the model has no critical points and the Gibbs random fields are multiple for all $K \in \mathbb{R}$. For $p=\psi\left(L_{*}\right)$, we have $t=9 / 5$ and $x_{*}^{(1)}=x_{*}^{(2)}=3$, which corresponds to $K_{*}=(\ln 3) / 4$. In this case, $x_{k} \rightarrow 3$ if $K \leqslant K_{*}$, and $x_{k} \rightarrow+\infty$ if $K>K_{*}$.

\section{Concluding remarks}

\subsection{Construction and structural properties}

The graphs introduced in this paper are constructed from motifs $M_{1}, \ldots, M_{5}$ in an algorithmic way, like the hierarchical lattices introduced in [13], and employed in [16] where they were supplied with long-range (decorating) bonds. However, our main construction principle - to replace nodes with the graphs of the previous level — differs from the one used in those papers, where the graphs of the previous level replaced the bonds. Hierarchical graphs of this kind model the real world networks with the so-called modular structure, which are organized in tightly knit communities with relatively sparse connections between them. For more details herein, we refer the reader to [29, 30] and to the publications cited therein. In contrast to the graphs introduced in [16], see also [29], our decorated graphs are not scale-free. For them, the node degree distribution is of intermediate type such that all the moments exist. The corresponding characteristic function can be extended to a meromorphic function, analytic in the complex neighborhood of zero, see (18), whereas for scale-free graphs such functions are nonanalytic at zero. All our graphs are amenable, which correlates with the property just discussed, see (14), (9), and (19). An interesting property is that, like for the graphs studied in [16], adding decorations forms the graphs possessing the small-world property.

\subsection{Critical points}

The appearance of critical points, and hence of phase transitions, for the Ising model confirms good communicating properties of the underlying graphs. For our graphs, in contrast to the hierarchical lattices for which phase transitions take place even without decorations, critical points can exist only if the decorations are applied, with any $p>0$. The most interesting related fact is that for the graph based on $M_{1}$, the antiferromagnetic Ising model has no phase transitions for any $L$ and $p$. This is due to the generic frustration of such models - the elementary factor $R_{k}(a, b, c)$ which appears in (35) with negative $L$ cannot be maximized. We expect that the same is true for the graphs based on $M_{5}$. To check if this conjecture of ours is true we plan to study a Potts model on the same graph and $q \geqslant 3$, for which such a frustration is no longer actual. We also plan to 
look for critical points with nonzero $h$, as well as for critical points of the Ising model with the underlying graphs based on the remaining motifs. However, the corresponding problems are much tougher, so that the appropriate numerical means should be applied.

\section{Acknowledgement}

The authors benefited from fruitful discussions on the matter of this work held with our colleagues Yuri Kondratiev and Vasyl Ustimenko for which they are cordially grateful. The authors are also grateful to the unnamed referee whose remarks and suggestions were helpful in improving the presentation of the article.

\section{References}

1. Erdős P., Rényi A., Publicationes Mathematicae, 1959, 6, 290.

2. Erdős P., Rényi A., Publications of the Mathematical Institute of the Hungarian Academy of Sciences, 1960,5, 17.

3. Białas, P., Burda, Z., Wacław, B. Causal and homogeneous networks. In: Science of complex networks, 14,AIP Conf. Proc., 776, Amer. Inst. Phys., Melville, NY, 2005.

4. Watts D.J., Strogatz H., Nature, 1998, 393, 440; doi:10.1038/30918

5. Barabási A.-L., Albert R., Science, 1999, 286, 509; doi:10.1126/science.286.5439.509

6. Milo R. et al., Science, 2002, 298, 824; doi:10.1126/science.298.5594.824

7. Itzkovitz S. et al., Phys. Rev. E, 2003, 68, 026127; doi:10.1103/PhysRevE.68.026127

8. Alon U., Science, 2003, 301, 1866; doi:10.1126/science.1089072 Nature Reviews Genetics, 2007, 8, 450; doi: $10.1038 / \mathrm{nrg} 2102$.

9. Kashtan N., Itzkovitz S., Milo R., Alon U., Phys. Rev. E, 2004, 70, 031909; doi:10.1103/PhysRevE.70.031909

10. Itzkovitz S., Alon U., Phys. Rev. E, 2005, 71, 026117; doi:10.1103/PhysRevE.71.026117

11. Matias C. et al, REVSTAT, 2006, 4, 31.

12. Häggström O., Adv. Appl. Probab., 2000, 32, 39.

13. Griffiths R.B., Kaufman M., Phys. Rev. B, 1982, 26, 5022; doi:10.1103/PhysRevB.26.5022

14. Bleher P.M., Žalys E., Litovsk. Mat. Sb., 1988, 28, 252 [English translation in Lithuanian Math. J., 1989, 28, 127; doi:10.1007/BF01027189.

15. Bleher P.M., Žalys E., Commun. Math. Phys., 1989, 120, 409; doi:10.1007/BF01225505

16. Hinczewski M., A. Nihat Berker, Phys. Rev. E, 2006, 73, 066126; doi:10.1103/PhysRevE.73.066126

17. Wróbel M., Condens. Matter Phys., 2008, 11, 341.

18. Kotorowicz M., Albanian Journal of Mathemathics, 2008, 3, 235.

19. Barlow M.T. Heat kernels and sets with fractal structure. In: Heat kernels and analysis on manifolds, graphs, and metric spaces (Paris, 2002). Contemp. Math., 338, Amer. Math. Soc., Providence, RI, 2003.

20. Newman M.E.J., SIAM Review, 2003, 45, 167; doi:10.1137/S003614450342480

21. Ruelle D., Statistical mechanics: Rigorous results. W.A. Benjamin, Inc., New York-Amsterdam, 1969.

22. Erdős P., Kleitman D.J., Rothschild B.L., Asymptotic enumeration of $K_{n}$-free graphs. In: Colloquio Internazionale sulle Teorie Combinatorie (Rome, 1973), Tomo II, pp. 19. Atti dei Convegni Lincei, No. 17, Accad. Naz. Lincei, Rome, 1976.

23. Futorny V., Ustimenko V., Acta Appl. Math., 2007, 98, 47; doi:10.1007/s10440-007-9144-8

24. Barrat A., Weigh M., Eur. Phys. J. B, 2000, 13, 547; doi:10.1007/s100510050067

25. Georgii H.-O., Gibbs measures and phase transitions. de Gruyter Studies in Mathematics, 9. Walter de Gruyter \& Co., Berlin, 1988.

26. Kępa D., Kozitsky Y., Condens. Matter Phys., 2008, 11, 313.

27. Jonasson J, Steif J.E., J. Theoret. Probab., 1999, 12, 549; doi:10.1023/A:1021690414168

28. Bovier, A., Statistical mechanics of disordered systems. A mathematical perspective. Cambridge Series in Statistical and Probabilistic Mathematics, Cambridge University Press, Cambridge, 2006; doi:10.1017/CBO9780511616808.004.

29. Hinczewski M., Phys. Rev. E, 2007, 75, 06611104; doi:10.1103/PhysRevE.75.061104

30. Kashtan N., Mayo A.E., Kalisky T., Alon U., PLoS Comput. Biol., 2009, 5, e1000355; doi:10.1371/journal.pcbi.1000355 


\title{
Іерархічні випадкові графи з мотивів: структурні властивості
} та критичні точки моделі Ізінга

\author{
Моніка Которович, Юрій Козицький \\ Інститут математики, Університет Марії Кюрі-Склодовської, Люблін
}

Вводиться і вивчається клас випадкових графів, збудованих в алгоритмічний спосіб з п'яти мотивів, знайдених у [Milo R., Shen-Orr S., Itzkovitz S., Kashtan N., Chklovskii D., Alon U., Science, 2002, 298, 824]. Конструкційна схема нагадує схему, застосовану у [Hinczewski M., A. Nihat Berker, Phys. Rev. E, 2006, $\mathbf{7 3}, 066126]$, згідно з якою короткосяжні ребра є невипадкові, тоді як довгосяжні ребра виникають незалежно і з однаковою ймовірністю. Описано ряд структурних властивостей графів, серед яких $€$ розподіл ступенів, кластерність, аменабільність, властивість тісного світу. Для одного з мотивів вивчається критична точка моделі Ізінга, визначеної на відповідному графі.

Ключові слова: аменабільність, розподіл ступенів, кластерність, граф тісного світу, модель Ізінга, критична точка 